\title{
Computer-Assisted TURP Training and Monitoring
}

\author{
M.P.S.F. Gomes and B.L. Davies \\ Mechatronics in Medicine Laboratory, Department of Mechanical Engineering, \\ Imperial College of Science, Technology and Medicine, Exhibition Road, \\ London SW7 2BX, UK \\ $\{$ p.gomes, b.davies\}@ic.ac.uk \\ http://www.me.ic.ac.uk/case/mim/
}

\begin{abstract}
A computer-assisted surgical trainer for Transurethral Resection of the Prostate (TURP) is described. The surgeon resects a rubber prostate phantom using real surgical tools, under endoscopic visual guidance. Additionally, a computer display provides navigational information and shows the progression of the resection. The core of the trainer consists of a low-cost PC and a commercial optical tracker. The extension of the trainer to the operating room, to be used as an in vivo monitoring system, is proposed. This novel two-stage approach aims to bridge the gap between training on a simulator and performing a real procedure in the operating room.
\end{abstract}

\section{Introduction}

There is an increasing need for validated training aids for endoscopic surgery that do not require the presence of a qualified surgeon, the involvement of a patient, or the use of live animals [1-12]. One of the key requirements of minimally invasive endoscopic procedures is the surgeon's ability to locate anatomical features in 3D space usually hidden behind an access port, and to position tools in specific locations whilst avoiding others. This requires mastering the anatomy, as well as an ability to visualise a 3D mental picture of it, from a combination of theoretical knowledge, experience, preoperative examinations, ultrasound scans, and a localised view given by an endoscopic camera.

In the specific case of Transurethral Resection of the Prostate, the goal is to use a minimally invasive resectoscope to remove adequate prostatic tissue, without resecting distal to the verumontanum, damaging the ureteric orifices, or perforating the capsule (Fig.1).

The traditional training follows the typical pattern of i) observing an experienced surgeon doing a number of procedures, and ii) performing a number of procedures, closely monitored by an experienced surgeon. Courses are conducted at the Royal College of Surgeons of England, London, where trainee urological surgeons can resect a prostate phantom but this is usually a one-off session and not all trainees attend.

The urologist conventionally carries out a TURP using a resectoscope inserted through the urethra. The resectoscope (Fig.3) performs three functions:

1. It allows the urologist to visualise the area to be resected (the prostatic urethra).

This is accomplished with the help of a high intensity light source, a camera and a 
series of lenses. The camera is usually linked to a monitor for more convenient visualisation (Fig.2).

2. It resects prostatic tissue, by utilising a wire loop through which a high frequency AC current is passed. A different waveform is used to coagulate bleeding arteries and veins.

3. It has a separate channel for irrigation with a sterile hypo-osmolar solution. Irrigation is used to improve the viewing of the operating field.

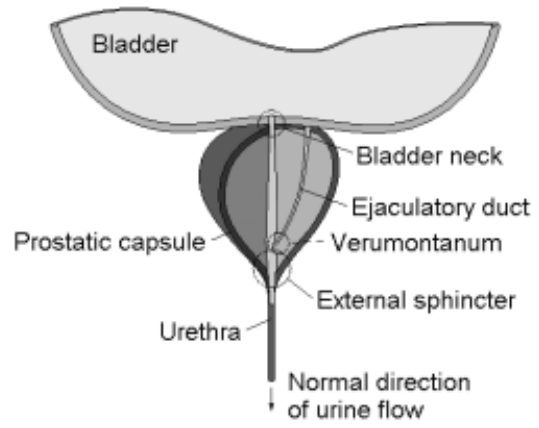

Fig. 1. Anatomy of the prostate gland

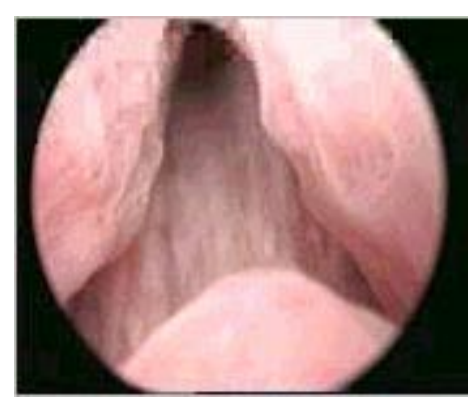

Fig. 2. Endoscopic view of the prostate gland, showing the verumontanum and the bladder neck

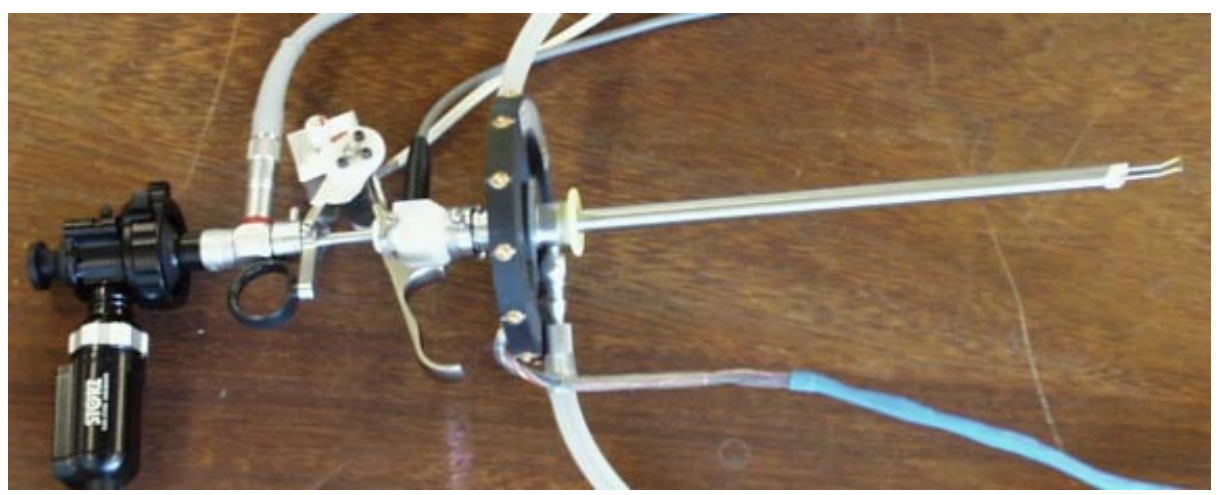

Fig. 3. Resectoscope elements (the ring and the potentiometer arrangement attached to the working element have been specifically developed for the trainer)

The main objectives of the computer-assisted TURP trainer presented are:

- To provide a realistic hands-on trainer.

A physical phantom of the prostate gland, of similar "feel" to real tissue, is resected, using very similar surgical equipment and procedure as those used in the 
operating room. The nature of the procedure, and the way it is perceived, must not be significantly affected by the trainer.

- To provide objective data to enable measurement of surgical skills and performance.

The amount of tissue resected, perforation or damage to high-risk areas, and the time taken to complete the procedure can all be logged.

- To provide extra guidance for navigation in 3D space.

A computer graphical interface is provided that depicts a scaled $3 D$ model of the prostate phantom, with the current position of the resectoscope superimposed in real time, so that the trainee always knows the position of the cutting loop within the prostate. Visual warnings are also given when the loop is close to high-risk areas. The important anatomical landmarks and the tissue resected are also shown, allowing the progress of the resection to be monitored.

\section{Materials and Methods}

The system comprises a mock-up abdomen (a perspex box with a rubber membrane) and a rubber bladder. For each resection, a rubber prostate is inserted into the rubber bladder and clamped. The box has an outlet for the irrigant and sits in a tray, in a supporting fixture. The position and orientation of the box, and hence of the phantom, is monitored using a camera-based tracker. The artificial prostate is resected by the surgeon, using a resectoscope instrumented for tracking. Two screens are provided, one depicting the endoscopic view, the second depicting 3D navigational information in relation to the prostate and the resectoscope. A snapshot of the trainer in operation is given in Fig. 4.

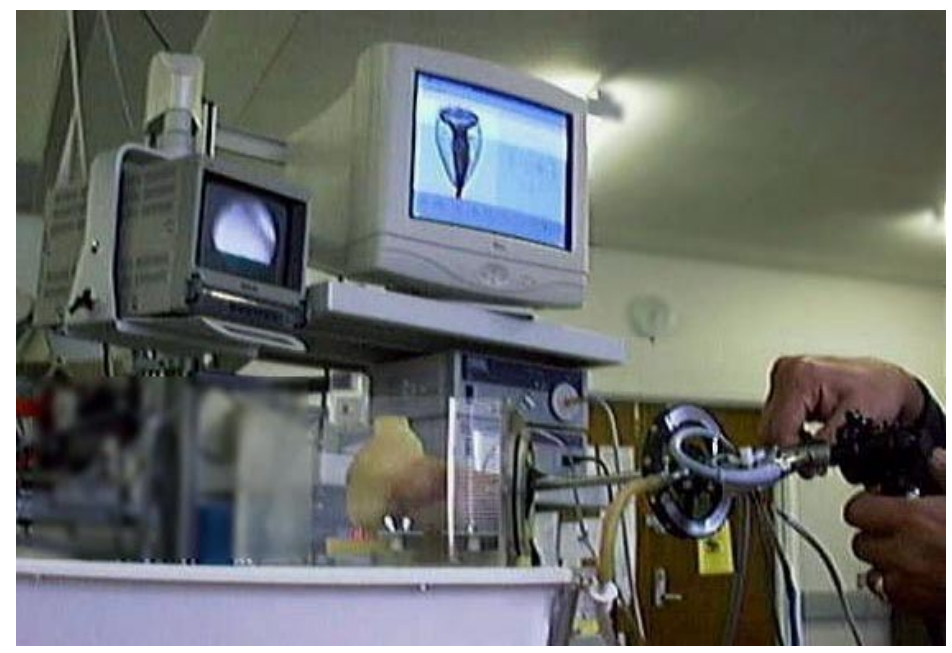

Fig. 4. Computer-assisted TURP trainer in use. The optical tracker is is mounted on a tripod and cannot be seen in this image. 


\subsection{Instrumentation}

Resectoscope motion The resectoscope's position and orientation is tracked using a purpose-built IRED ring clamped to its sheath. A camera-based system (Flashpoint 5000, Image Guided Technologies Inc., Colorado, USA) is used to track the $100 \mathrm{~mm}$ diameter ring, with 12 equally spaced IREDs (Fig.5). This novel IRED tool is necessary because the resectoscope can be rotated about the axis of its sheath by more than $\pm 180^{\circ}$.

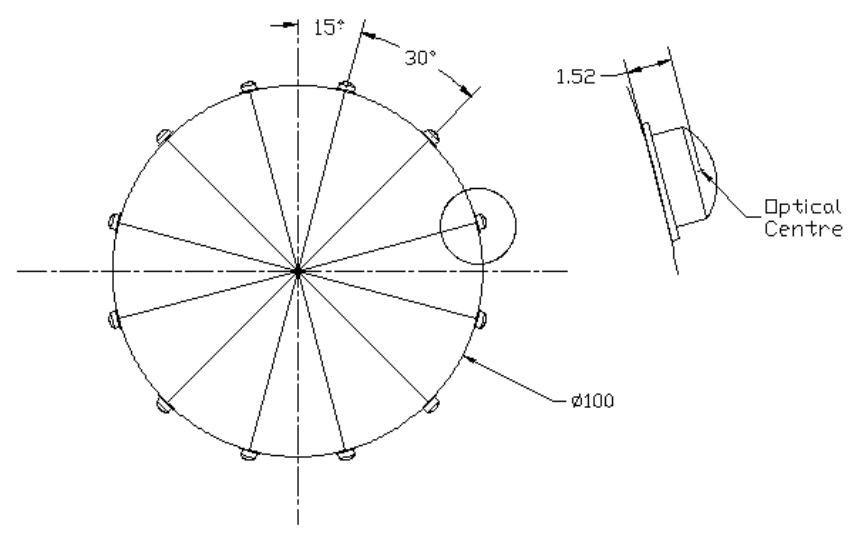

Fig. 5. IRED arrangement for optical tracking

A tool definition file is supplied to the optical tracker, containing the relative positions of all the IREDs and the tool tip. During the operation, the optical tracker calculates the tool tip's location from the positions of the visible IREDs.

There are many constraints to the shape of the ring, e.g. it has to allow the use of a conventional resectoscope and hence allow the operation of the irrigation inlet and outlet taps; it cannot obstruct the surgeon's movements or interfere with the procedure; it should not significantly change the "feel" of the tool; it has to be sterilisable; and it should also be easily adapted to standard instruments. The current design complies with these constraints.

A Dynamic Reference Frame (a special IRED probe, used as the origin of a coordinate system for the readings from the optical tracker) is attached to the perspex box, in a fixed location. This makes the readings, provided by the camera-based tracking system, independent from the position of the camera sensor.

Cutting loop travel The prostate phantom is resected by an electrical cutting loop. The loop moves along the inside of the resectoscope shaft, in a linear path of $25 \mathrm{~mm}$ extension. The resected chip separates from the rest of the phantom when the cutting loop fully enters the resectoscope's body. The surgeon moves the loop in and out of the resectoscope by operating a standard "scissor grip" on the resectoscope handle. 
A potentiometer has been coupled to the resectoscope's trigger by a gear mechanism to track the cutting loop travel. The potentiometer is connected to the low cost PC by means of an A/D board, using a cable with detachable plugs, similar to those for the IRED ring.

Diathermy activation The cutting action of the diathermy loop is activated by pressing a foot pedal connected to the diathermy unit. This action is sensed by a switch attached to the foot pedal and this is used to appropriately modify the computer display. The switch is connected to the PC, via the same data acquisition A/D board.

\subsection{Software}

Platform The software for the trainer has been developed using Microsoft Visual $\mathrm{C}++$, Microsoft Foundation Classes, and OpenGL, for the Windows 9x platform. The software runs on a Pentium III $500 \mathrm{MHz}$ machine.

Graphical user interface Whilst operating conventionally, the surgeon views the endoscopic camera display, and uses both hands to operate the resectoscope and ancillary equipment, as well as one foot to activate the diathermy unit. For the trainer, extra intervention or information has to be kept simple and to a minimum. Essentially, what the computer display of the trainer provides is i) information about the resectoscope's position in three-dimensional space, and ii) information about the tissue already resected.

In order to convey information in a quick and simple way, different views can be shown (Fig.6):

1. A 3D rendering of the model and of the resectoscope. The user can decide between displaying the whole model or displaying a partial view by cutting away the top part, which lies above the resectoscope.

2. A $2 \mathrm{D}$ view of the section through the cutting loop position, perpendicular to the base of the model, showing the model's outline, the resectoscope's position, and the area resected.

3. A collection of $2 \mathrm{D}$ views (similar to that described in (b) above), shown as thumbnail images, at pre-specified positions, equally spaced between the bladder neck and the verumontanum.

The user can chose which views are shown at any one time, as well as wireframe or solid models. The display of the resectoscope changes colour to provide simple and immediate visual feedback about the state of the instrument (green in normal mode; red if cutting; grey if obscured). The background of the $2 \mathrm{D}$ enlarged view also changes from light grey to yellow or red if the resectoscope's tip is too close to the bladder neck of the verumontanum, respectively. 


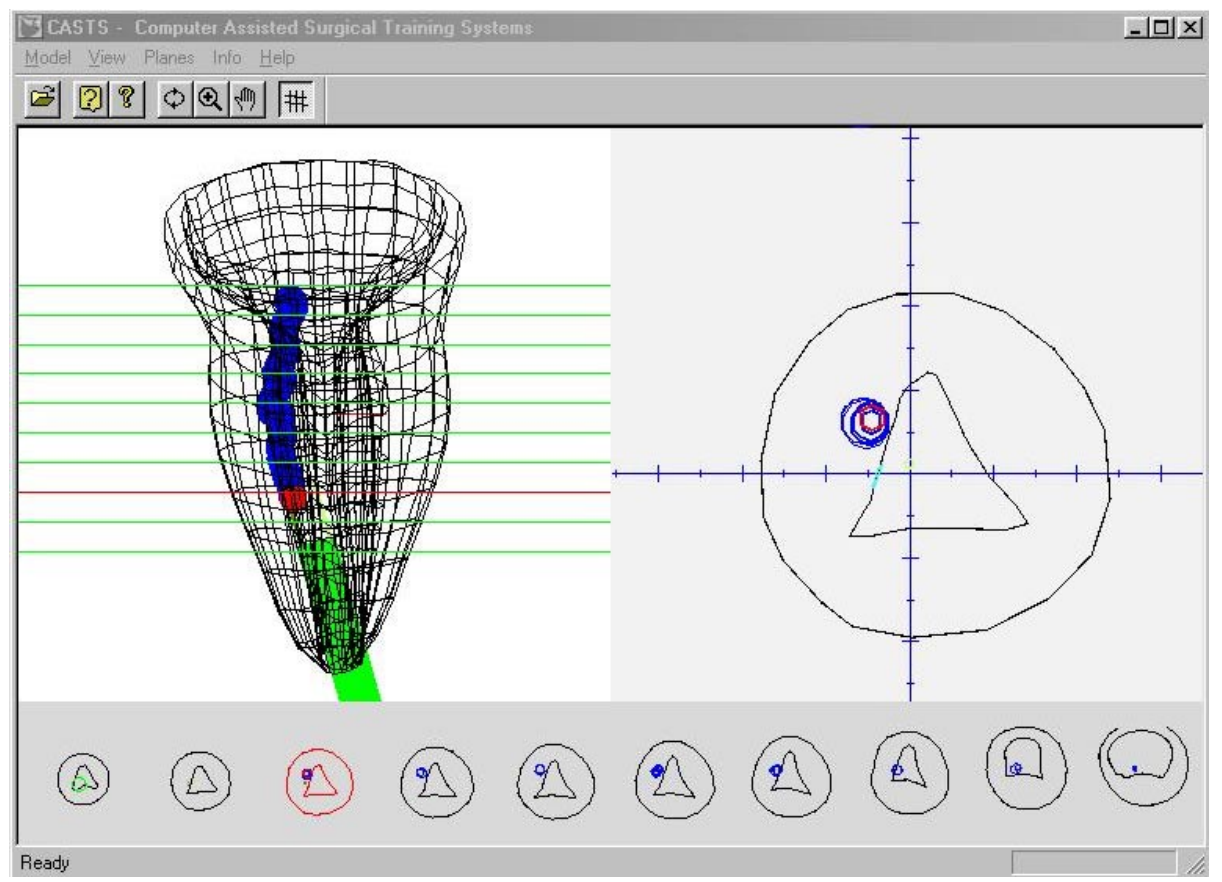

Fig. 6. The graphical user interface

Model representation The graphics model of the prostate phantom is a set of NURBS (Non-Uniform Rational B-Splines), stored in the IGES (Initial Graphics Exchange Specification) standard format, and loaded at the start-up of the trainer's software.

The data for the model was obtained by measuring hard casts of the rubber prostate, using the optical tracker, and fitting NURBS to these points, using Rhino (Robert McNeel and Associates, Washington, USA).

Cuts representation A separate graphical model is created, containing the cuts made on the phantom, without changing the original prostate model. This allows clear visualisation of the resected material and the progress of the resection.

Soft tissue deformation During a resection, the phantom moves and deforms, just like the real prostate, by the compressing and cutting action of the resectoscope, in a way related to the nature of the phantom's material and the phantom's fixture. In order to provide a realistic graphical display of this deformation, the NURBS control points are displaced and rendered. The displacements are precalculated and stored in look-up tables for speed.

Deformations caused by prolonged cutting and swelling due to the absorption of the irrigant are also observed. Clamps are used to partially constrain these 
deformations and make them predictable without detracting from the realistic 'feel' of the prostate which moves, in vivo, within the pelvic girdle attachments.

\section{Discussion and Conclusions}

Preliminary tests by surgeons have shown the in vitro system to be realistic and a useful addition to training. The computer display provides additional visual confirmation of the resectoscope cutting relative to the critical prostatic features. The use of a resectable physical phantom of the prostate provides a realistic sense of "feel" on the handle of the resectoscope when pressing and cutting the prostate. This approach has been found to provide a simpler and lower-cost system than using a totally virtual reality (VR) system in which the haptic sense at the tool handle is not provided by resecting a physical phantom but is provided by a complex series of force profiles applied to the tool using motorised torque control.

However, several problems have been found which need tackling before widespread use of the trainer can be beneficial. Inaccuracies of several milimeters have been observed when the traditional resectoscope's cutting loop bends and also when the phantom swells by absorbing irrigant. Also, with the progress of the resection, the system slows down due to increased graphical rendering (of the cuts model) and some cuts made are not recorded or are displayed late. This however can be compensated for, to some degree, by increased computing costs.

The solution to improve accuracy lies in closing the loop, i.e., in verifying what tissue is actually resected. This can be accomplished by using ultrasound to image the resection. However, this would change the procedure and, therefore, would not comply with our first objective listed in section 1. Additionally, the current phantom cannot be imaged with ultrasound and an alternative, which would still provide similar "feel" to human tissue, would have to be found. Ideally, the phantom would be non-absorbent and made to tighter manufacturing tolerances.

For the proposed in vivo monitoring system (Fig.7), pre-operative transrectal ultrasound scans of the patient's prostate (taken just before the resection procedure) are to be used to build up a model of the specific 3D anatomy. Per-operative rectal ultrasound scanning, using an ultrasound probe fitted with infra-red emitter diodes (IREDs), allows the progress of the resection to be monitored during the procedure.

This attempt to improve the quality of prostatectomy training has given a number of insights into the problems of CAS (Computer-Assisted Surgery) in vitro trainers for general use in soft tissue surgery. It is felt that the ability to link the in vitro approach to an in vivo training and monitoring system is potentially rewarding. The need to bridge the wide gap between simple training systems and patient trials by trainees is of great importance.

\section{Acknowledgements}

The collaboration of Mr. A.G. Timoney, from the Department of Urology, Southmead Hospital, Bristol, United Kingdom, and of Mr. P.V.S. Kumar, from the Taunton and 
Somerset Hospital, United Kingdom, is gratefully acknowledged. This project is sponsored by the Engineering and Physical Sciences Research Council (EPSRC) of the UK. Karl Storz Endoscopy (UK) has kindly loaned medical equipment. Limbs \& Things (UK) provided moulds for the prostate model.

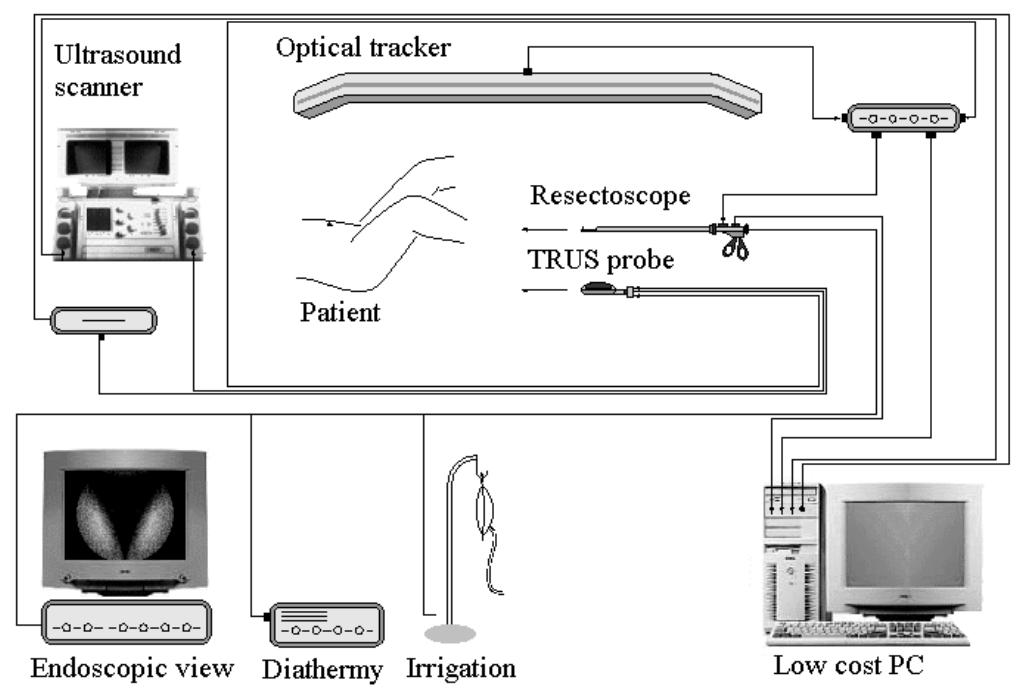

Fig. 7. The proposed in vivo TURP monitor

\section{References}

1. http://www.cine-med.com/

2. http://www.ht.com/products_endosim.htm

3. http://www.vrweb.com/WEB/DEV/MEDICAL.HTM\#MIST

4. Ayache, N., Cotin, S., Delingette, H., Clement, J. M., Y.Russier and Marescaux, J. (1998) Simulation of endoscopic surgery, Minimally Invasive Therapy \& Allied Technologies 7 (2), 71-77

5. Bauer, A., Soldner, E. H., Ziegler, R. and Muller, W. (1995) Virtual reality in the surgical arthroscopic training, Second Annual International Symposium on Medical Robotics and Computer Assisted Surgery, Baltimore, Maryland, USA, 350-355

6. Gomes, M. P. S. F., Barrett, A. R. W., Timoney, A. G. and Davies, B. L. (1999) A computerassisted training/ monitoring system for TURP - Structure and design, IEEE Transactions on Information Technology in Biomedicine 3 (4), 242-251

7. Kleinszig, G., Radetzky, A., Auer, D. P., Pretschner, D. P. and Auer, L. M. (1998) ROBOSIM: Simulation of Endoscopic Surgery, Society for Minimally Invasive Therapy 10th Anniversary International Conference, London

8. Logan, I. (1995) Virtual environment knee arthroscopy training system, http://www.enc.hull.ac.uk/CS/VEGA/medic/surgery.html 
9. McCarthy, A. D. and Hollands, R. J. (1998) A commercially viable virtual reality knee arthroscopy training system, Medicine Meets Virtual Reality: 6, San Diego, USA, 302-308

10. Schill, M.A., Wagner, C., Hennen, C., Bender, H-J. and Manner, R. (1999) EyeSi - A simulator for intra-ocular surgery, Medical Image Computing and Computer-Assisted Intervention - MICCAI'99, Cambridge, UK, 1166-1174

11. Szekely, G., Brechbuhler, C., Hutter, R., Rhomberg, A. and Schmid, P. (1998) Modelling of soft tissue deformation for laparoscopic surgery simulation, Medical Image Computing and Computer-Assisted Intervention - MICCAI'98, Boston, USA, 550-561

12. Xuan, J., Wang, Y., Sesterhenn, I. A., Moul, J. W. and Mun, S. K. (1998) 3-D model supported prostate biopsy simulation and evaluation, Medical Image Computing and Computer-Assisted Intervention - MICCAI'98, Boston, USA, 358-367 